\title{
二氧化碳诱导的可逆离子液体中钯纳米 颗粒的合成及应用研究
}

刘帅帅 ${ }^{1,2}$, 张兆富 $^{1^{*}}$, 印帅 ${ }^{1}$, 刘会贞 ${ }^{1,2}$, 马珺 ${ }^{1}$, 侯民强, 樊红雷 ${ }^{1}$, 韩布兴 ${ }^{1,2^{*}}$

1. 中国科学院化学研究所, 北京分子科学国家研究中心, 胶体界面与化学热力学重点实验室, 北京 100190

2. 中国科学院大学化学与化工学院, 北京 100049

*通讯作者, E-mail: zhangzf@iccas.ac.cn; hanbx@iccas.ac.cn

收稿日期: 2021-04-12; 接受日期: 2021-05-12; 网络版发表日期: 2021-09-17

国家重点研发计划(编号: 2017YFA0403101, 2017YFA0403003, 2017YFA0403103)资助项目

摘要本文采用二氧化碳诱导的1,8-二氮杂二环十一碳-7-烯(DBU)和醇溶液形成的可逆离子液体为介质, 通过 热还原的方法制备了金属钯纳米颗粒，研究了醇的链长对粒子尺寸的影响. 通过透射电子显微镜(TEM)和同步辐 射小角X射线散射(SAXS)原位表征等手段对纳米颗粒的形貌和粒径等进行了表征. 研究发现, 在没有二氧化碳时, DBU与醇组成的溶液中合成的钯呈块状; 在加入二氧化碳形成的离子液体中合成的钯纳米颗粒粒径均匀, 分散性 好, 粒子尺寸与离子液体中醇的链长关系不大. TEM表征得到的钯纳米粒子尺寸明显大于SAXS原位表征得到的 离子液体中粒子的尺寸, 说明在分离过程中钯粒子发生团聚现象. 此外, 我们研究了该液体体系中金属钯纳米颗 粒催化对甲基硝基苯和邻硝基苯甲醛加氢反应. 结果表明, 钯纳米颗粒对硝基的还原具有较高的催化活性, 且在 醛基存在时可以对硝基进行定向加氢。

关键词离子液体, 二氧化碳, 钯催化, 硝基苯还原

\section{1 引言}

2005年, Jessop 等 ${ }^{[1]}$ 报道了二氧化碳诱导下形成的 一种可逆离子液体, 随着二氧化碳的吸收与释放, 液体 的极性发生明显变化，溶液可以在非离子态与离子状 态间切换. 在他们的报道中, 向等摩尔 1,8 -二氮杂二环 十一碳-7-烯(1,8-diazabicyclo[5.4.0]undec-7-ene, DBU) 和正己醇的混合溶液中通入二氧化碳气体可以转换成 离子液体; 此离子液体可通过室温下通入氮气或者加 热的方式回到非离子状态(图1). 在这之后，许多关于
二氧化碳作为诱导剂的研究逐渐报道 ${ }^{[2]}$. 二氧化碳作 为一种智能小分子, 被应用于许多响应性材料, 包括 表面活性剂 ${ }^{[3,4]}$ 、水添加剂 ${ }^{[5]}$ 、亲疏水溶剂 ${ }^{[6,7]}$ 、可逆 离子液体 ${ }^{[8]}$ 、二氧化碳响应的聚合物 ${ }^{[011]}$ 等. 通过二氧 化碳的诱导, 这些材料具有了可调控的性能, 如从没有 表面活性到具有表面活性、从中性到离子性、从疏水 性到亲水性、从低极性到高极性.

二氧化碳诱导型离子液体在很多方面的应用已有 研究 ${ }^{[12,13]}$, 包括绿色溶剂 ${ }^{[14]}$ 、生物柴油的萃取 ${ }^{[15]}$ 和微 生物中碳氢化合物的提取 ${ }^{[16]}$ 等. 由于其溶剂性质可逆

引用格式: Liu S, Zhang Z, Yin S, Liu H, Ma J, Hou M, Fan H, Han B. Synthesis of palladium nanoparticles in carbon dioxide induced reversible ionic liquids and their application. Sci Sin Chim, 2021, 51: 1435-1441, doi: 10.1360/SSC-2021-0086 


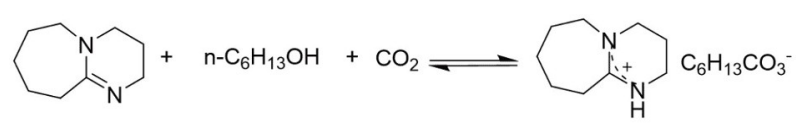

图 1 二氧化碳诱导DBU和正己醇的离子化过程

Figure 1 Ionization of DBU and 1-hexanol in the presence of carbon dioxide.

性, 有望应用于合成和分离的过程中, 通过调节溶剂的 性质减少溶剂的分离和替换步骤. 离子液体作为一种 绿色溶剂，已经广泛应用于金属纳米颗粒的合成过程 中 $^{[17]}$. 利用可逆离子液体的特性将金属纳米颗粒的合 成与催化性能研究相结合以减少金属纳米颗粒的分离 过程. 将可逆离子液体应用于金属纳米颗粒合成的研 究尚未见报道. 本工作使用可逆离子液体, 以金属钯 盐为前驱体, 同时通入二氧化碳和加热, 通过热还原 的方法得到了钯金属纳米颗粒.

苯胺, 特别是含有官能团的苯胺及其衍生物在农 药、制药、颜料、染料、精细化学品、材料和生命 科学等领域具有重要的应用 ${ }^{[18]}$. 传统的合成方法主要 是基于相关硝基化合物的还原反应. 非催化性的还原 反应需要使用化学当量的还原剂, 如硫、金属锌或铁 等, 这些方法往往会产生大量的废水废酸和杂质, 从 而导致一系列的环境问题. 使用氢气催化还原对于生 产苯胺类产物具有环境友好和高效等特点 ${ }^{[19]}$. 对于含 有取代基的硝基苯化合物还原得到相应的苯胺化合 物是一难题 ${ }^{[20,21]}$. 已有报道将金属Pd用于该还原反

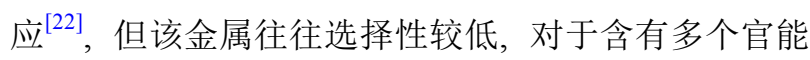
团的底物效果较差. 我们将原位得到的金属钯纳米颗 粒用于温和条件下硝基化合物的还原反应，发现其不 仅具有较高的催化活性，且对于含醛基的底物具有较 好的选择性.

\section{2 实验部分}

\section{1 试剂与仪器}

1,8-二氮杂二环十一碳-7-烯(DBU, 99\%)、1-丙 醇 $(99.9 \%) 、 1$-戊醇 $(99 \%) 、$ 正辛醇 $(98 \%)$ 、邻硝基 苯甲醛(98\%)购于Alfa Aesar化学试剂有限公司. 正 丁醇 $(99 \%)$ 、正己醇 $(98 \%)$ 、正庚醇 $(99 \%)$ 、醋酸钯 (98\%)、对甲基硝基苯(99\%)、十二烷(99\%)购于百 灵威试剂有限公司。甲醇、乙醇购于北京国药试剂 有限公司。二氧化碳( $99.999 \%) 、$ 氢气( $99.99 \%)$ 购于
北京分析仪器厂. 所有试剂直接使用, 未进一步 处理.

透射电子显微镜(TEM)采用JEOL-1011拍摄, 小角 $\mathrm{X}$ 射线散射(SAXS)于北京同步辐射光源(BSRF) $1 \mathrm{~W} 2 \mathrm{~A}$ 小角散射站测试, 气相色谱仪(GC-Agilent 6820, FID检 测器)用于还原反应产物的定量分析.

\section{2 二氧化碳诱导型离子液体([DBUH][C $\mathrm{C}_{x} \mathbf{H}_{2 x+1^{-}}$ $\left.\mathrm{CO}_{3}\right]$ )的合成}

将二氧化碳气体持续通入等摩尔DBU和醇的混 合溶液中, $3 \mathrm{~h}$ 后溶液呈乳白色黏稠状. 我们将使用不 同直链醇得到的离子液体 $[\mathrm{DBUH}]\left[\mathrm{C}_{x} \mathrm{H}_{2 x+1} \mathrm{CO}_{3}\right]$ 命名为 $\mathrm{C} x, x$ 表示体系中使用的直链醇的碳原子数.

\section{3 离子液体 $\mathbf{C} x$ 中钯金属纳米颗粒的合成}

在 $25 \mathrm{~mL}$ 干燥的单口瓶中加入 $10 \mathrm{~mL}$ DBU和醇 的等摩尔混合溶液, 加入约 $22 \mathrm{mg}$ 醋酸钯, 充分摚拌 至醋酸钯完全溶解. 向溶液中通入二氧化碳气体, 约 $3 \mathrm{~h}$ 后得到淡黄色黏稠液体. 取 $2.5 \mathrm{~mL}$ 该液体加至 $15 \mathrm{~mL}$ 反应釜中, 密封充入二氧化碳约 $2 \mathrm{MPa}$. 空气浴 $100^{\circ} \mathrm{C}$ 加热 $12 \mathrm{~h}$, 得到了分散在离子液体中的钯纳米 颗粒. 在此过程中, 醇起到还原剂的作用 ${ }^{[23 \sim 25]}$. 将在 不同直链醇形成的离子液体中得到的金属钯颗粒命 名为 $\mathrm{Pd} @ \mathrm{C} x$.

\section{4 金属钯颗粒的表征}

(1) 小角X射线散射表征

使用SAXS技术研究了分离前离子液体中金属钯 纳米颗粒的状态及粒径. 该检测在BSRF小角X射线散 射实验站 $(1 \mathrm{~W} 2 \mathrm{~A})$ 下进行. 使用的X射线波长为 $1.54 \AA$, 样品到检测器的距离为 $1.574 \mathrm{~m}$. 使用 CCD (Chargecoupled device, Mar 165 CCD) 探测器以获得需要的图片.

(2) 透射电子显微镜

将离子液体中的金属钯颗粒经多次乙醇洗涤、离 心过程以除去DBU和醇, $60^{\circ} \mathrm{C}$ 真空干燥 $12 \mathrm{~h}$. 金属钯颗 粒的形貌和粒径经透射电子显微镜(TEM)表征.

(3) X射线光电子能谱

X射线光电子能谱(XPS)用ESCALab 250Xi多功 能 $\mathrm{X}$ 射线光电子谱仪检测, 使用 $\mathrm{Al} \mathrm{K} \alpha$ 射线 $(\lambda=$ $1486.8 \mathrm{eV})$ 作为辐射光源. 结合能用C $1 \mathrm{~s}$ 峰 $(284.8 \mathrm{eV})$ 校正. 


\section{5 金属钯纳米颗粒催化硝基苯类化合物加氢}

以二氧化碳诱导的DBU和正己醇混合溶液形成 的离子液体中得到的金属钯纳米颗粒 $(\mathrm{Pd} @ \mathrm{C} 6)$ 为催化 剂, 研究了在混合溶液体系中的催化性能. 首先将得到 的含金属钯纳米颗粒的离子液体缓慢加热除去溶液中 的二氧化碳. 待体系降至室温后直接加入 $2 \mathrm{mmol}$ 对甲 基硝基苯/邻硝基苯甲醛，充入一定压力的氢气，使用 空气浴加热至 $80^{\circ} \mathrm{C}$ 或者室温下搅拌 $12 \mathrm{~h}$. 反应结束后 冷却至室温，缓慢放出残存的气体，加入一定量的正 十二烷作为内标，加入过量乙醇稀释，反应液经离心 获得上清液，使用气相色谱仪对甲基硝基苯及其产物 和邻氨基苯甲醛及其产物定量分析.

\section{3 结果与讨论}

\section{1 金属钯的合成与表征}

在不同醇体系的离子液体中通过热还原的方法合

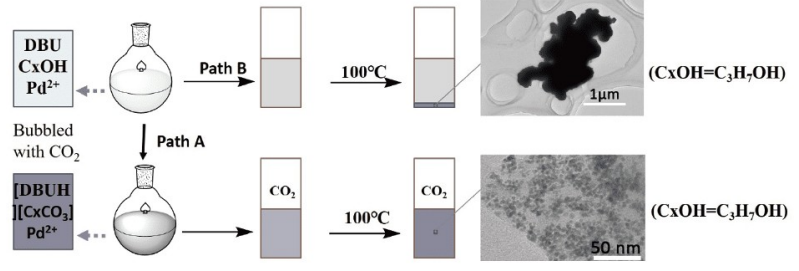

图 2 使用和不使用二氧化碳的条件下合成金属钯颗粒

Figure 2 Synthesis of Pd particles with or without the modification of $\mathrm{CO}_{2}$

成了金属钯纳米颗粒 $\mathrm{Pd} @ \mathrm{C} x$ (Path A, 图2), 同时在不 含二氧化碳的混合溶液中使用热还原的方法得到了金 属钯颗粒(Path B, 图2).

如图2所示, 在二氧化碳诱导型离子液体中得到的 金属钯在溶液中呈较好的分散状态。TEM研究表明, 金属钯颗粒呈均匀的球状，其粒径约5.0 6.7 nm (图 3a h)，粒子尺寸与离子液体中醇的链长关系不大; 而 在不使用二氧化碳的混合溶液中得到的是大块的金属
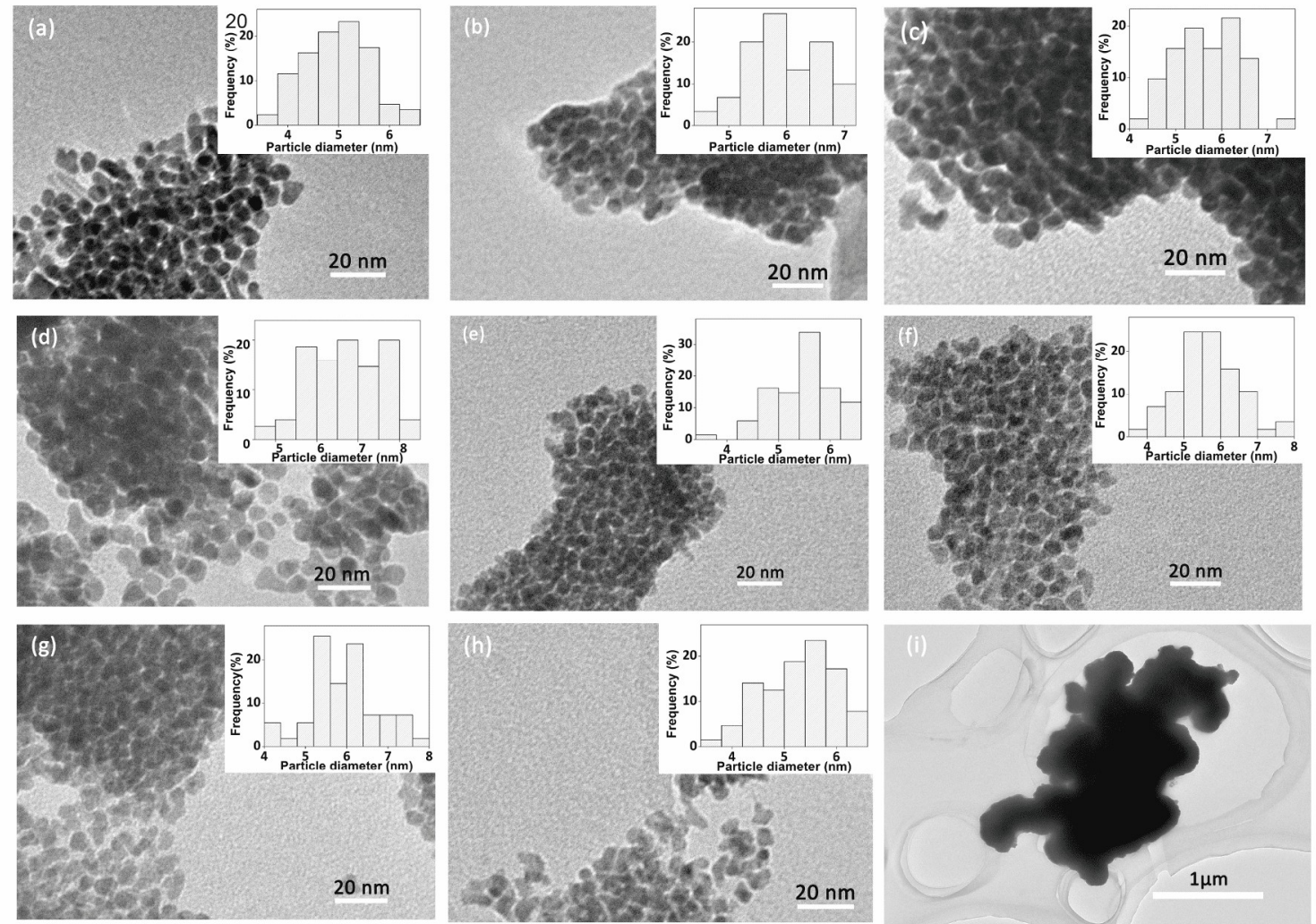

图 $3(\mathrm{a} \sim \mathrm{h}) \mathrm{Pd} @ \mathrm{C} x$ 的TEM电镜图, 其中 $\mathrm{C} x$ 分别为(a) C1, (b) C2, (c) C3, (d) C4, (e) C5, (f) C6, (g) C7 和(h) C8. 插图为相应的粒 径分布图. (i) DBU和己醇混合溶液中得到的钯颗粒TEM图

Figure 3 (a-h) TEM images of Pd@Cx. (a) C1, (b) C2, (c) C3, (d) C4, (e) C5, (f) C6, (g) C7, (h) C8. The insets show the size distribution of Pd nanoparticles. (i) TEM images of Pd particles prepared in the mixture of DBU and 1-hexanol. 

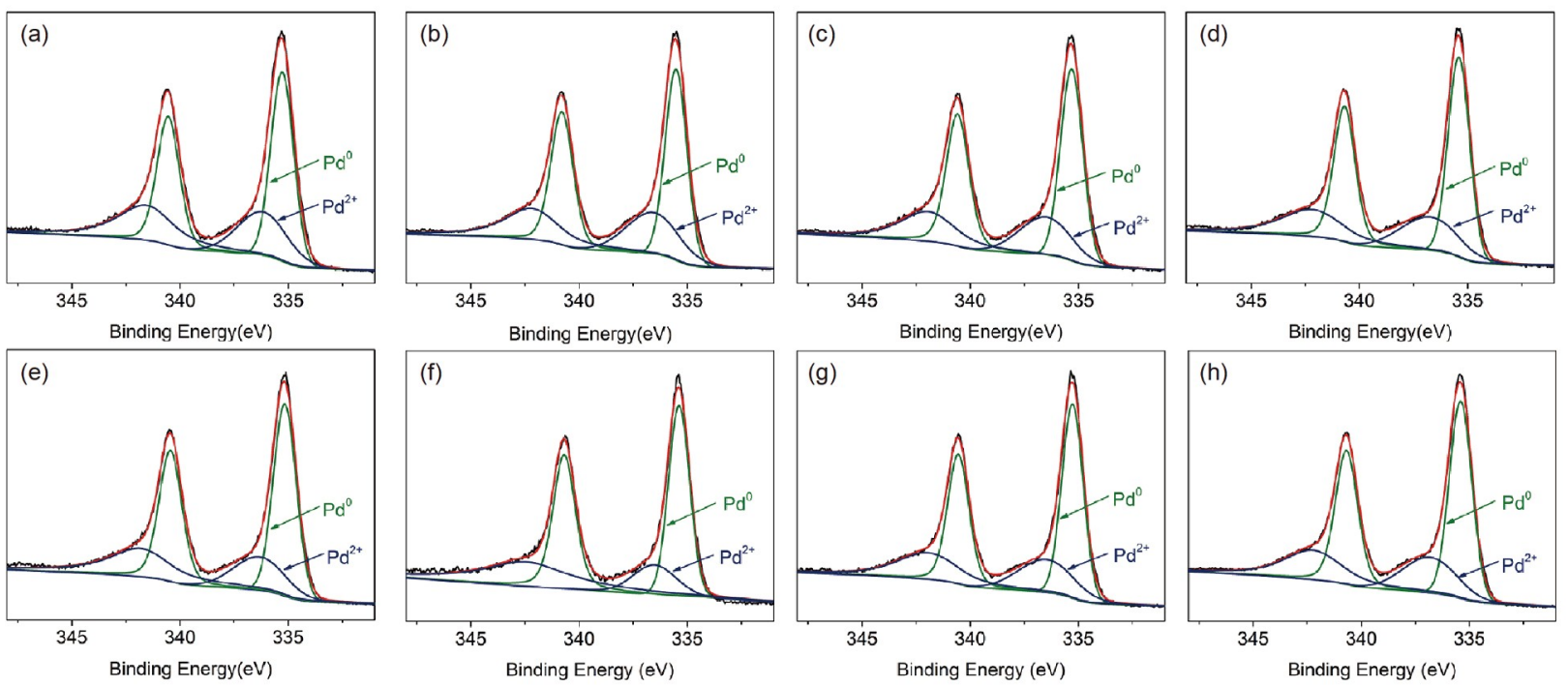

图 4 二氧化碳诱导型离子液体中制备的金属钯纳米颗粒中Pd 3d的XPS谱图. (a) C1; (b) C2; (c) C3; (d) C4; (e) C5; (f) C6; (g) C7; (h) C8 (网络版彩图)

Figure 4 Pd 3d XPS spectra for the Pd@Cx. (a) C1; (b) C2; (c) C3; (d) C4; (e) C5; (f) C6; (g) C7; (h) C8 (color online).

钯，且颗粒大部分沉降在反应釜底部，TEM表征说明 钯颗粒出现明显的聚集和结块(图3i). 这表明, 使用二 氧化碳诱导型离子液体热还原得到的金属钯具有较好 的分散性, 不容易发生聚集且颗粒粒径较小, 说明二氧 化碳在金属钯颗粒的形成过程中起到关键的作用。主 要原因之一是, 没有二氧化碳时, 混合溶液的黏度很 低, 导致金属团聚. 二氧化碳存在时, 离子液体的黏度 大, 从而抑制了钯粒子的团聚.

我们用XPS对钯纳米粒子的价态进行了表征. 金 属Pd 3d轨道价态分峰结果表明, 纳米颗粒表面中Pd价 态有两种, 零价钯和二价钯(图4), 其中二价钯的含量 分别为 $39.5 \% 、 37.9 \% 、 35.0 \% 、 35.5 \% 、 36.0 \%$ 、 $37.0 \% 、 35.5 \% 、 35.0 \%$, 这说明, 在分离和表征过程中 $\mathrm{Pd}$ 部分氧化, 这与文献结论 ${ }^{[26,27]}$ 一致.

为研究分离过程对钯纳米粒子尺寸的影响, 我们 还使用同步辐射SAXS技术对在正戊醇、正己醇、正 庚醇、正辛醇所形成的离子液体中制备的金属钯颗粒 进行了原位表征. 通过对其散射强度曲线拟合分析可 知，在溶液中的金属钯颗粒呈球状均匀分布，其粒径 均约 $4 \mathrm{~nm}$ (图5), 尺寸明显小于通过TEM得到的颗粒 粒径大小 $(5.0 \sim 6.7 \mathrm{~nm})$. 其中原因之一是 TEM表征前需 要经过金属钯纳米颗粒洗涤和离心的过程, 这些过程 中发生颗粒聚集从而粒径变大.

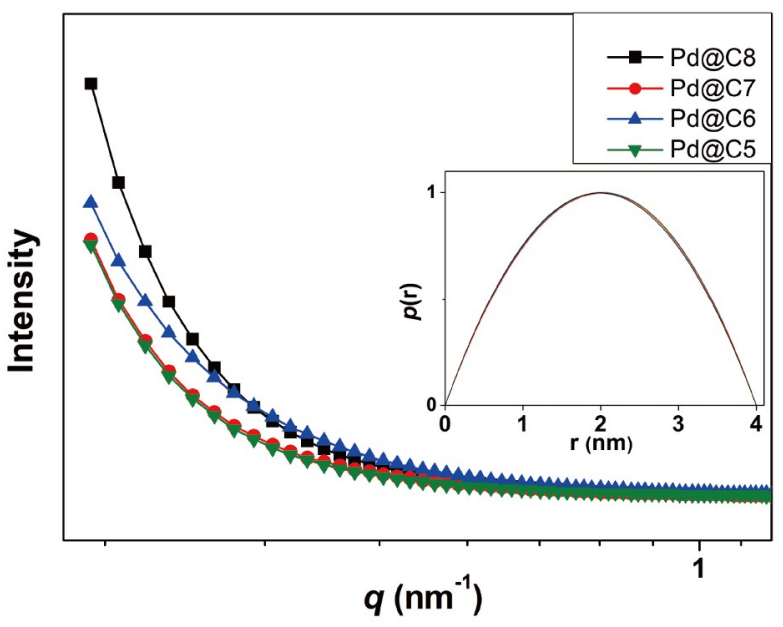

图 5 不同合成介质中钯纳米颗粒的 SAXS曲线. 插图为相 应的距离分布函数 $p(r)$ (网络版彩图)

Figure 5 SAXS profiles and pair-distance distribution function curves (the inset) of Pd nanoparticles (color online).

\subsection{Pd@C6的催化性能}

我们对不同醇合成的金属钯催化剂性能进行了考 察, 在使用 $2 \mathrm{MPa}$ 氢气还原对硝基甲苯的反应中, 正己 醇中制备的金属钯的催化效果最优，其次分别为正庚 醇、正戊醇，对甲基苯胺的产率分别为 $61.5 \%$ 、 $56.7 \% 、 55.3 \%$. 这说明溶剂中醇的链长对反应有明显 
影响. 我们进一步研究了Pd@C6的催化性能. 实验结 果如图6所示. 在对甲基硝基苯为底物的还原反应中 (图6a), 随着氢气压力的增加, 反应底物的转化率和产 物对甲基苯胺的产率都明显增高, 压力达 $4 \mathrm{MPa}$ 时, 对 硝基甲苯几乎全部被还原为对甲基苯胺(转化率 $>99.9 \%$, 产率 $>99.9 \%$ ). 在室温条件下以邻硝基苯甲醛 为底物的还原反应中(图6b), 随着氢气压力的增加, 邻 氨基苯甲醛的产率增加且无其他相应醛基还原产物生 成. 在 $2 \mathrm{MPa}$ 压力下，延长反应时间至 $6 \mathrm{~h}$ ，反应产率高 达 $99.7 \%$. 结果表明, 该催化体系具有较好的硝基化合 物的催化还原活性; 在醛基存在下, 对硝基可以实现定 向加氢. 我们对比了多种催化剂在对硝基苯甲醛还原 反应的催化效果, 发现该结果优于大部分文献 ${ }^{[28 ~ 31]}$ 中 报道的结果且催化条件相对温和(图6c).

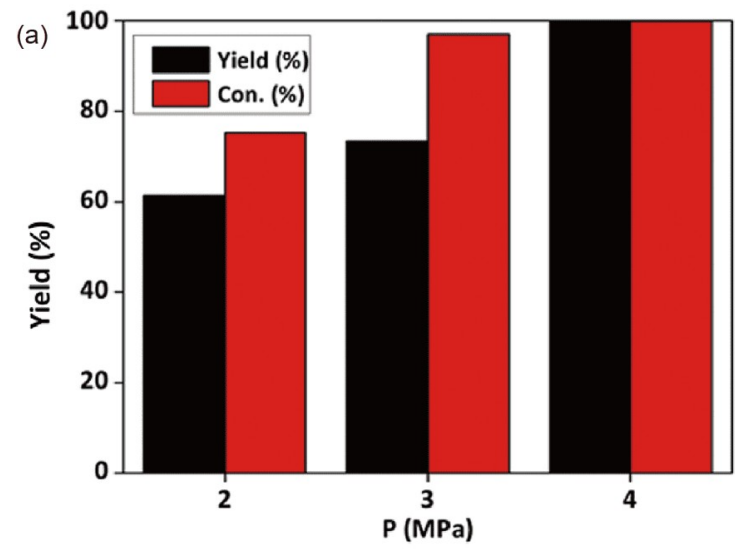

\section{4 结论}

通过热还原的方法在二氧化碳诱导型离子液体中 合成了金属钯纳米颗粒，TEM和SAXS表征表明，金属 钯纳米颗粒呈球状均匀分布, 粒径较小, 对照实验说明 二氧化碳在金属钯颗粒的形成过程中起着关键作用. 另外，TEM表征得到的钯纳米粒子尺寸明显大于 SAXS 原位表征得到的离子液体中粒子的尺寸，这说 明在钯纳米粒子分离过程中, 钯粒子发生团聚现象. 由此可以推测，通常用TEM表征得到的金属纳米粒子 尺寸可能大于分离前溶液中纳米粒子尺寸．对于硝基 苯类化合物的还原反应，离子液体中合成的金属钯纳 米颗粒表现出良好的催化活性，并且在醛基的存在下 可以实现硝基的定向加氢还原.

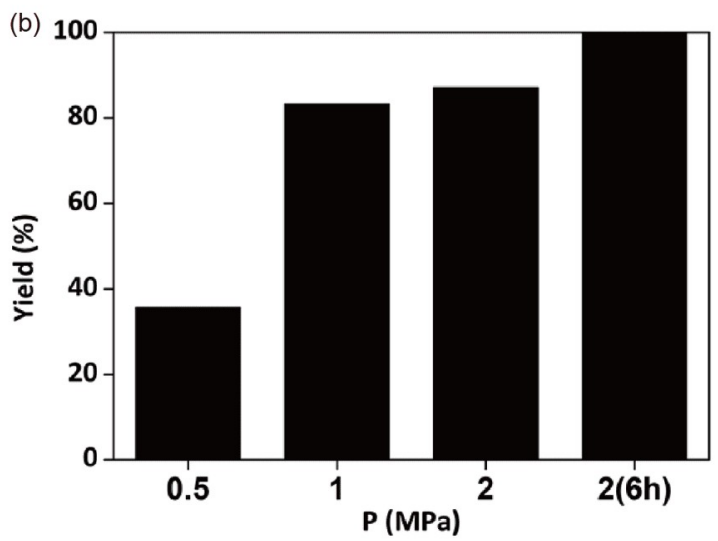

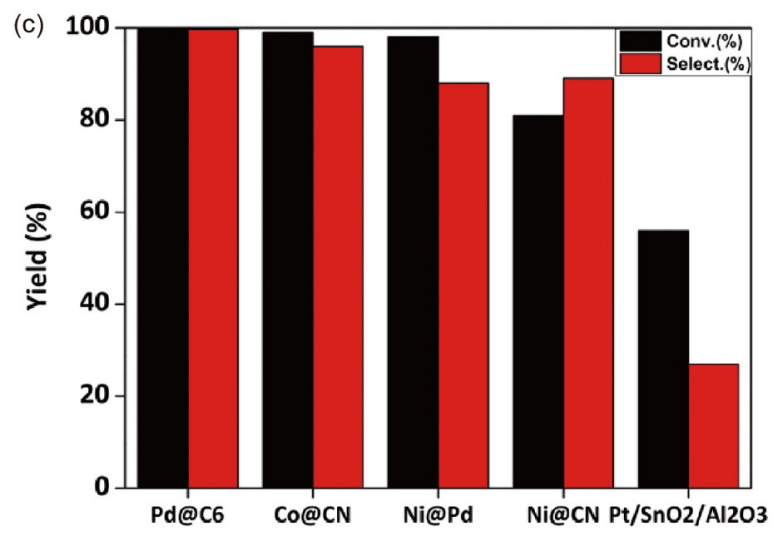

图 6 (a) 对甲基硝基苯的转化率和相应苯胺的产率. 反应条件: $2 \mathrm{mmol}$ 对甲基硝基苯, $2.5 \mathrm{~mL} \mathrm{Pd} @ \mathrm{C} 6,80^{\circ} \mathrm{C}$, 反应时间 $12 \mathrm{~h}$.

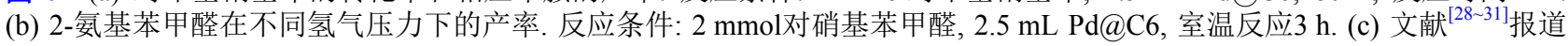
的2-氨基苯甲醛的转化率和选择性(网络版彩图)

Figure 6 (a) The conversion of $p$-nitrotoluene and yield of $p$-touidine. Reaction conditions: $2 \mathrm{mmol} p$-nitrotoluene, $2.5 \mathrm{~mL} \mathrm{Pd} @ \mathrm{C} 6,80{ }^{\circ} \mathrm{C}, 12 \mathrm{~h}$. (b) The yield of 2-aminobenzaldehyde in different $\mathrm{H}_{2}$ pressures. Reaction conditions: 2 mmol 2-nitrobenzaldehyde, $2.5 \mathrm{~mL} \mathrm{Pd} @ \mathrm{C} 6$, room temperature, $3 \mathrm{~h}$. (c) The conversion and selectivity of 2-aminobenzaldehyde using various catalysts referring to Refs. [28-31] (color online). 


\section{参考文献}

1 Jessop PG, Heldebrant DJ, Li X, Eckert CA, Liotta CL. Nature, 2005, 436: 1102

2 Yang Z, He C, Sui H, He L, Li X. J $\mathrm{CO}_{2}$ Utilization, 2019, 30: 79-99

3 Xu P, Wang Z, Xu Z, Hao J, Sun D. J Colloid Interface Sci, 2016, 480: 198-204

4 Liu D, Suo Y, Tan J, Lu H. Soft Matter, 2017, 13: 3783-3788

5 Mercer SM, Robert T, Dixon DV, Chen CS, Ghoshouni Z, Harjani JR, Jahangiri S, Peslherbe GH, Jessop PG. Green Chem, 2012, 14: 832-839

6 Pollet P, Eckert CA, Liotta CL. Chem Sci, 2011, 2: 609-614

7 Jessop PG, Phan L, Carrier A, Robinson S, Dürr CJ, Harjani JR. Green Chem, 2010, 12: 809-814

8 Yamada T, Lukac PJ, Yu T, Weiss RG. Chem Mater, 2007, 19: 4761-4768

9 Darabi A, Jessop PG, Cunningham MF. Chem Soc Rev, 2016, 45: 4391-4436

10 Garcia-Valdez O, Brescacin T, Arredondo J, Bouchard J, Jessop PG, Champagne P, Cunningham MF. Polym Chem, 2017, 8: 4124-4131

11 Yu B, Zhao Y. Polym Chem, 2017, 8: 4132-4139

12 Jessop PG, Mercer SM, Heldebrant DJ. Energy Environ Sci, 2012, 5: 7240-7253

13 Phan L, Chiu D, Heldebrant DJ, Huttenhower H, John E, Li X, Pollet P, Wang R, Eckert CA, Liotta CL, Jessop PG. Ind Eng Chem Res, 2008, 47: 539-545

14 Jessop PG. Green Chem, 2011, 13: 1391-1398

15 Mohammad Fauzi AH, Amin NAS. Renew Sustain Energy Rev, 2012, 16: 5770-5786

16 Samorì C, Torri C, Samorì G, Fabbri D, Galletti P, Guerrini F, Pistocchi R, Tagliavini E. Bioresource Tech, 2010, 101: 3274-3279

17 Luska KL, Migowski P, Leitner W. Green Chem, 2015, 17: 3195-3206

18 Doherty S, Knight JG, Backhouse T, Bradford A, Saunders F, Bourne RA, Chamberlain TW, Stones R, Clayton A, Lovelock K. Catal Sci Technol, 2018, 8: 1454-1467

19 Formenti D, Ferretti F, Scharnagl FK, Beller M. Chem Rev, 2019, 119: 2611-2680

Zhang S, Chang CR, Huang ZQ, Li J, Wu Z, Ma Y, Zhang Z, Wang Y, Qu Y. J Am Chem Soc, 2016, 138: 2629-2637

Salahshournia H, Ghiaci M. Appl Organomet Chem, 2019, 33: 13

Arai N, Onodera N, Dekita A, Hori J, Ohkuma T. Tetrahedron Lett, 2015, 56: 3913-3915

3 Hirai H, Chawanya H, Toshima N. Reactive Polymers, 1985, 3: 127-141

Roucoux A, Schulz J, Patin H. Chem Rev, 2002, 102: 3757-3778

Liu M, Zhang J, Liu J, Yu WW. J Catal, 2011, 278: 1-7

Xiong Y, Chen J, Wiley B, Xia Y, Yin Y, Li ZY. Nano Lett, 2005, 5: 1237-1242

Shen Y, Zhang S, Liao F, Sun J, Dang Q, Shao M, Kang Z. ChemCatChem, 2020, 12: 504-509

Sun X, Olivos-Suarez AI, Oar-Arteta L, Rozhko E, Osadchii D, Bavykina A, Kapteijn F, Gascon J. ChemCatChem, 2017, 9: 1854-1862

Jian S, Li Y. Chin J Catal, 2016, 37: 91-97

Pan H, Peng Y, Lu X, He J, He L, Wang C, Yue F, Zhang H, Zhou D, Xia Q. Mol Catal, 2020, 485: 110838

Liu M, Tang W, Xie Z, Yu H, Yin H, Xu Y, Zhao S, Zhou S. ACS Catal, 2017, 7: 1583-1591 


\title{
Synthesis of palladium nanoparticles in carbon dioxide induced reversible ionic liquids and their application
}

\author{
Shuaishuai Liu ${ }^{1,2}$, Zhaofu Zhang ${ }^{1 *}$, Shuai Yin ${ }^{1}$, Huizhen Liu ${ }^{1,2}$, Jun Ma ${ }^{1}$, Minqiang Hou ${ }^{1}$, \\ Honglei Fan ${ }^{1}$, Buxing Han ${ }^{1,2^{*}}$ \\ ${ }^{1}$ Beijing National Laboratory for Molecular Science, CAS Key Laboratory of Colloid and Interface and Chemical Thermodynamics, Institute of \\ Chemistry, Chinese Academy of Sciences, Beijing 100190, China \\ ${ }^{2}$ School of Chemistry and Chemical Engineering, University of Chinese Academy of Sciences, Beijing 100149, China \\ *Corresponding authors (email: zhangz@iccas.ac.cn; hanbx@iccas.ac.cn)
}

\begin{abstract}
We synthesized Pd particles in reversible ionic liquid formed by carbon dioxide induction of 1,8-diazabicyclo [5.4.0] undec-7-ene (DBU) and 1-hexanol solution. Transmission electron microscopy (TEM) and small-angle X-ray scattering (SAXS) were used to characterize their morphology and size. The particles have smaller size and more uniform size distribution compared with those synthesized in the mixed solution without carbon dioxide. The particles size obtained by TEM is obviously larger than that obtained by SAXS, which indicates the aggregation of the Pd particles in the separation and washing processes of TEM characterization. Then we studied the catalytic performance of Pd nanoparticles in p-nitrotoluene and 2-nitrobenzaldehyde hydrogenation reaction. The results showed that the nanoparticles have high catalytic activity and selectivity for nitro group with aldehyde group unchanged.
\end{abstract}

Keywords: ionic liquid, carbon dioxide, Pd catalysis, nitrobenzene reduction

doi: $10.1360 /$ SSC-2021-0086 